\title{
Creencias sobre las causas de la hipertensión arterial: influencia en las estrategias de afrontamiento y el estilo de vida
}

\author{
Beliefs about the Causes of Hypertension: Influence on Coping \\ Strategies and Lifestyle
}
Silvia Deborah Ofman ${ }^{1}$ Redalyc - Carolina Iris Pereyra Girardi ${ }^{2}$ Redalyc - Pedro Cófreces ${ }^{3}$ - Dorina Stefani ${ }^{4}$ Redalyc
1,2,3,4 Instituto de Investigaciones Cardiológicas ININCA/UBA-CONICET

Argentina

Fecha correspondencia:

Recibido: noviembre 9 de 2015.

Aceptado: agosto 12 de 2016.

\section{Forma de citar:}

Deborah, S., Pereyra, C., Cófreces P., \& Stefani, D. (2016). Creencias sobre las causas de la hipertensión arterial: influencia en las estrategias de afrontamiento y el estilo de vida. Rev. CES Psicol., 9(2),114-127.

\section{Open access}

(c) Copyright

Licencia creative commons

Etica de publicaciones

Revisión por pares

Gestión por Open Journal System

ISSN: 2011-3080

DOI: http://dx.doi.org/10.21615/

cesp.9.2.8

Sobre los autores:

1. Doctora en el área de Humanidades Médicas. Licenciada en Psicología (UBA). Becaria

Postdoctoral del Consejo

Nacional de Investigaciones

Científicas y Técnicas (CONICET).

\section{Resumen}

El objetivo del presente estudio es comparar las estrategias de afrontamiento y el estilo de vida relacionado con la salud en un grupo de pacientes hipertensos, según el tipo de creencias que presentan dichos pacientes en relación a las causas de la enfermedad. El diseño es descriptivo-comparativo, de corte transversal. Participaron 200 pacientes hipertensos adultos, de nivel socioeconómico medio, habitantes de la Ciudad Autónoma de Buenos Aires (Argentina). Se utilizaron un cuestionario sociodemográfico y una entrevista semiestructurada, elaborados ad hoc; Ways of Coping Questionnaire y Health-Promoting Lifestyle Profile II. En el grupo de pacientes con creencias de tipo psicosocial se observó más una estrategia de afrontamiento por la cual el individuo reconoce haber influido en las causas del problema. En cambio, el grupo con creencias de tipo biológicas se focalizó en atender la enfermedad.

Palabras clave: Actitudes, Creencias, Hipertensión arterial, Estrategia de Afrontamiento, Estilo de vida.

\section{Abstract}

This study aims to compare coping strategies and lifestyle related to health, in a group of hypertensive patients, depending on the type of beliefs they have in regards to the causes of the disease. The design is descriptivecomparative, cross sectional. 200 hypertensive adult patients, ranked in middle economic status, living in Buenos Aires city (Argentina) participated in the study. Both ad hoc, sociodemographic questionnaire and a semistructured interview instruments were administered, as well as Ways of Coping Questionnaire and the Health-Promoting Lifestyle Profile II. A coping mechanism of admitting to have influenced in the causes of the disease was observed among the group of patients with psychosocial beliefs. Instead of the group of biological beliefs, that was focused on finding a solution for the disease.

Keywords: Attitudes, Beliefs, Hypertension, Coping Behavior, Lifestyle. 
Pág 115

Miembro del Laboratorio de Psicología Social y Salud del Instituto de Investigaciones Cardiológicas ININCAI CONICETUBA.

2. Doctora en el área de Humanidades Médicas. Licenciada en Psicología (Universidad del Salvador). Becaria Postdoctoral del CONICET. Miembro del Laboratorio de Psicología Social y Salud del ININCA/CONICETUBA.

3. Licenciado en Ciencias de la Comunicación (UBA). Personal Técnico Profesional del CONICET. Miembro del Laboratorio de Psicología Social y Salud del ININCA/CONICET-UBA.

4. Doctora en el área de Salud Mental. Licenciada en Sociología (UBA). Investigadora Principal del CONICET. Jefa del Laboratorio de Psicología Social y Salud del ININCA/CONICETUBA.

\section{Introducción}

El marco conceptual clásico desarrollado por Ajzen y Fishbein (1980) sostiene que las actitudes se conforman por tres componentes: cognitivo, afectivo y conductual. Las creencias, en tanto elementos cognitivos de las actitudes, son aquellas que determinan en el individuo la predisposición a actuar de determinada manera. En el campo de la salud, una de las articulaciones de dicha interrelación se ve reflejada en el hecho de que las creencias de los individuos tienen efectos sobre los diversos comportamientos asociados al proceso de salud-enfermedad, tales como las conductas preventivas, la adherencia terapéutica, la interpretación de los síntomas, la búsqueda de ayuda médica, entre otros (Moreno \& Gil Roales-Nieto, 2003). A partir de estas conceptualizaciones, dentro del campo de la Psicología de la Salud se han utilizado diversos modelos (esquemas cognitivos, cognición de enfermedad, modelos de sentido común de la enfermedad, modelo de creencias en salud, locus de control en salud, entre otros), a fin de estudiar, explicar e intentar predecir, el proceso de la adherencia terapéutica y las diversas conductas en salud, en las enfermedades crónicas (Morrison \& Bennett, 2008; Ortiz Viveros \& Ortega Herrera, 2011).

Coincidiendo con este planteamiento, la teoría de Lazarus y Folkman (1986) afirma que las creencias son, entre otros, uno de los factores que influyen en el tipo de afrontamiento que presentan los sujetos ante una situación determinada de estrés, en este caso, en lo referente al ámbito de la salud-enfermedad. El afrontamiento representa, a diferencia de un comportamiento adaptativo automatizado, los esfuerzos que realiza de manera particular cada individuo, a fin de mitigar el impacto producido por los eventos estresantes (Leibovich de Figueroa \& Schufer, 2002). Entre los recursos que influyen en el modo en que el individuo afronta la enfermedad, se encuentra el repertorio de creencias que circula en una sociedad acerca de la misma. Es decir, que las atribuciones causales que elaboran las personas sobre los motivos de la enfermedad vienen determinadas, entre otros, por la cultura (Rodríguez Marin, Pastor, \& Lopez Roig, 1993).

Tal como se ha mencionado, las creencias acerca del proceso salud-enfermedad influyen en el estilo de vida, modulando los comportamientos tendientes a promover o conservar la salud (Pender, 1982). El estilo de vida se conforma por aquellos comportamientos habituales, cotidianos, que pueden tender a que el individuo mantenga la salud o, por el contrario, se exponga al riesgo de enfermar (Morales Calatayud, 1999). Estos patrones de comportamiento están determinados por la interrelación entre los factores personales, las interacciones sociales y las condiciones socioeconómicas y ambientales (Organización Mundial de la Salud [OMS], 1998).

Los esquemas o teorías implícitas sobre las creencias de una enfermedad presentan una organización similar, a través del tiempo y las culturas. Dichos esquemas se componen por las diversas dimensiones relacionadas con el desarrollo y evolución de las enfermedades: identidad, causas, consecuencias, período temporal, curación, control, gravedad, entre otros. La dimensión relativa a las causas que originaron la enfermedad es uno de los componentes principales de dichas representaciones, ya que suele ser el primer nivel explicativo al que hacen referencia los pacientes ante la vivencia de una enfermedad (León, Páez, \& Díaz, 2003).

Existen diferencias individuales en el contenido de estas creencias. Diferencias que están determinadas por diversos factores, tales como las características personales, el género, la cultura, las condiciones de vida, el acceso a la prestación en salud, la educación y otros. Las diversas identidades sociales que tienen los individuos, en 
Pág 116

Desde una perspectiva biopsicosocial, se la conceptualiza como una enfermedad multicausal, en la que confluyen estilos de vida, factores psicológicos y ambientales, entre otros, situación que complejiza la adecuada adherencia terapéutica (Cordero et al., 2011). función del contexto en el que se encuentren, juegan un papel importante al momento de percibir la enfermedad (Morrison \& Bennett, 2008).

La hipertensión arterial (HTA) es una de las enfermedades sobre las cuales se han estudiado los significados, representaciones y creencias que elaboran los pacientes sobre la misma, así como las estrategias de afrontamiento ante ella y la adherencia terapéutica (Álvarez, Rueda, González, \& Acevedo, 2010; Arrieta-Pérez \& Garfías-Jiménez, 2010; Bernal Trujillo, Lemos Hoyos, Medina Durango, Ospina Duque, \& Torres, 2009; Corugedo Rodríguez, Martín Alfonso, \& Bayarre Vea, 2011; Espinosa García, Martell Claros, Llerena Ruiz, \& Fernández Bergés Gurrea, 2012; García de Alba-García, Salcedo-Rocha, Vargas-Guadarrama, \& García de Alba-Verduzco, 2012; Guevara-Gasca \& Galán-Cuevas, 2010; Martín Alfonso et al, 2007; Silva et al., 2008).

Existen varias razones y fenómenos que hacen de la HTA un trastorno de creciente relevancia para el ámbito de la investigación en salud. Desde el punto de vista epidemiológico, en los últimos años se ha evidenciado una alta prevalencia a nivel mundial, situación a la cual se suma el hecho de que muchos sujetos la padecen y no son diagnosticados (OMS, 2013). Desde el aspecto fisiológico, la HTA presenta la característica de tratarse de un trastorno silente, crónico, y que constituye uno de los factores de riesgo más importantes para las enfermedades cardiovasculares (Gómez LLambí \& Piskorz, 2013). Por otra parte, desde una perspectiva biopsicosocial, se la conceptualiza como una enfermedad multicausal, en la que confluyen estilos de vida, factores psicológicos y ambientales, entre otros, situación que complejiza la adecuada adherencia terapéutica (Cordero et al., 2011).

A partir de lo expuesto, el objetivo del presente trabajo es comparar las estrategias de afrontamiento y el estilo de vida relacionado con la salud en un grupo de pacientes hipertensos, según el tipo de creencias que presentan dichos pacientes en relación a las causas de la enfermedad.

\section{Hipótesis}

La hipótesis del estudio sostiene que las estrategias de afrontamiento a la enfermedad y el estilo de vida relacionado con la salud difieren entre los pacientes hipertensos, en función del tipo de creencias sobre las causas de la enfermedad que ellos elaboran.

\section{Método}

\section{Diseño}

Se siguieron los pasos correspondientes al diseño descriptivo-comparativo según el tipo de creencia, de corte transversal.

\section{Participantes}

La muestra se conformó por 200 pacientes hipertensos adultos, de nivel socioeconómico medio, reclutados en distintos centros de salud públicos y privados, situados en la Ciudad Autónoma de Buenos Aires (Argentina).

La estrategia de muestreo fue no probabilística, de tipo intencional. Los criterios de inclusión que se siguieron fueron: presentar, al menos, un año de diagnóstico de HTA; realizar tratamiento médico para la HTA y firmar el consentimiento informado. 


\section{Instrumentos}

Cuestionario sociodemográfico: Con el fin de obtener el perfil sociodemográfico de la muestra, se elaboró un cuestionario ad hoc de preguntas abiertas y cerradas con alternativas fijas.

Entrevista semiestructurada: En el marco de la misma, elaborada ad hoc, se indagó a los pacientes respecto a las creencias sobre cuál es la principal causa atribuida a su HTA.

Ways of Coping Questionnaire (WCQ) [Cuestionario sobre Modos de Afrontamiento]: Para estudiar las estrategias de afrontamiento frente a la HTA, se aplicó la versión del WCQ (Edwards \& O 'Neill, 1998; Folkman, Lazarus, Pimley, \& Novacek, 1987) traducida al español (Lazarus \& Folkman, 1986).

El cuestionario consta de 32 ítems que definen las siguientes estrategias de afrontamiento: Resolución planificada de problemas (desarrollo de tácticas para solucionar el problema); Confrontación (esfuerzos por alterar la situación, acompañados de cierto grado de hostilidad y riesgo); Aceptación de la responsabilidad (reconocimiento del papel que ha tenido el sujeto en el origen y/ o mantenimiento del problema); Distancia (esfuerzos cognitivos que realiza el sujeto por apartarse o separarse del problema, intentando evitar que le afecte); Escape/Evitación (empleo de conductas motoras, tales como comer, dormir, consumir tóxicos, entre otras, a fin de no confrontarse con la problemática); Connotación/Reconsideración positiva (percepción de los posibles aspectos positivos que presenta o haya presentado el evento estresante); Autocontrol (esfuerzos por regular y controlar los sentimientos y respuestas emocionales) y Búsqueda de apoyo social (acudir a otras personas para buscar apoyo, consejo, comprensión, asesoramiento).

La aplicación consistió en solicitarle a los sujetos que respondieran con qué frecuencia (en una escala Likert de 1 a 5: 1=nunca, 2=pocas veces, 3=a veces sí y a veces no, $4=$ frecuentemente y $5=$ siempre) pensaron o se comportaron de la manera en que se indica en cada uno de los ítems. Se planteó a todos los pacientes que respondieran en función de la misma situación estresante: su condición de hipertenso.

El puntaje final en cada estrategia de afrontamiento osciló, en una escala tipo Likert, entre 1 (nunca) y 5 (siempre), y se obtuvo promediando los valores asignados a los ítems que las conforman. A mayor puntaje, mayor utilización de la estrategia.

Se halló una adecuada consistencia interna del instrumento para este tipo de estudio (Fleiss, Levin, \& Paik, 2004), obteniéndose un valor de Alpha de Cronbach $=.717$.

Health-Promoting Lifestyle Profile II (HPLP II) [Perfil de estilo de vida promotor de la salud II]: Con el fin de estudiar el estilo de vida relacionado con la salud, se seleccionó el cuestionario HPLP II (Walker, Sechrist, \& Pender, 1987), en su versión en español (Walker, Kerr, Pender, \& Sechrist, 1990).

El instrumento mide la frecuencia con la que los sujetos mantuvieron hábitos saludables, durante los últimos seis meses. Se compone de 52 ítems que conforman un puntaje total, que a su vez se agrupan en seis dimensiones: Autorrealización (evalúa el logro de propósitos y metas personales); Relaciones interpersonales (se centra en la comunicación y las relaciones con los otros); Nutrición (valora la elección de una dieta diaria saludable); Actividad física (grado de participación en actividades físicas 
leves, moderadas y enérgicas); Responsabilidad con la salud (dimensión relacionada con la detección temprana de síntomas y la búsqueda de ayuda profesional) y Manejo del estrés (evalúa la utilización de recursos psicológicos y físicos que ayuden a controlar o reducir la tensión diaria).

La aplicación consistió en solicitarle a los sujetos que indicaran la frecuencia con la cual realizaron cada conducta y hábito mencionado en los ítems. Para ello, debieron elegir una de las siguientes categorías, correspondientes a una escala Likert de 1 a 5: $1=$ nunca; $2=$ pocas veces; $3=a$ veces sí y a veces no; $4=$ frecuentemente; $5=$ siempre. El puntaje de cada dimensión resultó del promedio de los valores asignados a los ítems que la conforman. A mayor puntaje, el comportamiento es más saludable.

Se halló una adecuada consistencia interna del instrumento para este tipo de estudio (Fleiss et al., 2004), obteniéndose un valor de Alpha de Cronbach = .915.

\section{Procedimiento}

Luego de obtener la aprobación de las autoridades y los profesionales de las instituciones de salud, se sostuvo una entrevista individual con aquellos pacientes que decidieron participar en el estudio, el cual se encuentra adscripto al Subsidio CONICET PIP No 112200801 00114, dirigido por la Dra. Dorina Stefani. Previamente, se les explicó el objetivo de la investigación y se les solicitó la firma del consentimiento informado.

\section{Análisis de datos}

Con el fin de describir el perfil sociodemográfico de la muestra (sexo, edad, nacionalidad, estado civil, nivel de educación, religión, tipo de ocupación), se calcularon distribuciones de frecuencias, porcentajes, medias aritméticas y desvíos estándar, de acuerdo al nivel de medición de las variables.

Las respuestas recabadas en la entrevista semiestructurada se analizaron siguiendo los pasos del análisis temático (Souza Minayo, 1997), a partir del cual se elaboraron categorías teóricas y empíricas, que sintetizaron los temas emergentes: Creencias sobre las principales causas de la hipertensión: a) causas de tipo psicosocial y b) causas de tipo biológicas.

Para evaluar el afrontamiento y el estilo de vida relacionado con la salud, se calcularon las medias aritméticas y los desvíos estándar de las estrategias y dimensiones del WCQ y HPLPII, respectivamente.

Con el objeto de comparar estas variables entre los grupos con diferentes creencias sobre las causas de la enfermedad, se calculó un Análisis Multivariado de la Varianza (MANOVA) para dos factores: a) tipo de creencias sobre causas de la enfermedad y b) género. Se incluyó esta última categoría, a fin de controlar su probable influencia en el resto de las características del estudio, dada la importancia del género como variable sociocultural, en tanto se encuentra íntimamente relacionada con el fenómeno de la salud-enfermedad (Nerín, 2015; Ofman, Pereyra Girardi, Cófreces, \& Stefani, 2014).

Los datos fueron analizaron con el soporte técnico del software SPSS versión 18.0 (Castañeda, Cabrera, Navarro, \& de Vries, 2010). En todos los casos se consideró un valor de probabilidad de error igual o menor a 05. 
Pág 119

En lo que se refiere a las creencias sobre las causas de la HTA, a partir del análisis cualitativo de las respuestas consignadas en la entrevista semiestructurada, surgieron dos grupos de creencias: "causas de tipo psicosocial", que conformaron la mayoría de las respuestas $(63.5 \%)$ y "causas de tipo biológicas", referidas por el $36.5 \%$ de los pacientes.

\section{Resultados}

La muestra final quedó conformada por 189 sujetos (94.5\%), que son quienes refirieron algún tipo de causa para su HTA, mientras que los 11 restantes (5.5\%), respondieron desconocer cuál era el motivo de la enfermedad.

El perfil sociodemográfico de la muestra indica que la edad promedio de los pacientes fue de 56.2 años (de=7.9), siendo 94 varones y 95 mujeres. El 92\% era de nacionalidad argentina; el $66 \%$ estaba casado; y el 76\% profesaba la religión católica. En cuanto al nivel de escolaridad, el 49\% tenía formación terciaria o universitaria y el $40 \%$ refirió haber realizado estudios secundarios. Por otra parte, el $73 \%$ de la muestra se encontraba laboralmente activo al momento del estudio. Los principales tipos de ocupación de los pacientes fueron: empleado, profesional, comerciante, oficios varios y docente.

Teniendo en cuenta el Índice de Nivel Económico Social de Gino Germani (Grimson et al., 1972; Mora \& Araujo, 2002), se puede indicar que la muestra pertenece al nivel socioeconómico medio, debido al alto grado de educación que presenta y al tipo de ocupación que realiza. Además, a este dato se le agrega el hecho de que el $95 \%$ de los pacientes eran atendidos en obra social o prepaga, mientras que el $5 \%$ que concurría al hospital público, lo hacía en general por derivación del médico tratante de la obra social.

En cuanto al tiempo de diagnóstico de HTA, el 40\% respondió tener entre 1 y 5 años, otro 40\% entre 5 y 15 años, y el restante 20\% tenía más de 15 años de diagnóstico.

En lo que se refiere a las creencias sobre las causas de la HTA, a partir del análisis cualitativo de las respuestas consignadas en la entrevista semiestructurada, surgieron dos grupos de creencias: "causas de tipo psicosocial", que conformaron la mayoría de las respuestas (63.5\%) y "causas de tipo biológicas", referidas por el $36.5 \%$ de los pacientes.

La categoría "psicosocial" quedó conformada por aquellas creencias relacionadas con los denominados factores de riesgo modificables, los cuales se asocian a variables de tipo psicológicas y sociales, tales como: características de personalidad, conflictos vinculares, estrés laboral, estilos de vida, duelos, entre otros. Algunos ejemplos de este tipo de respuestas son: "Me enfermé por haber pedido aumento en el trabajo, después de diez años de no dármelo", "Mi presión es emotiva. La hipertensión la tengo encima", "Creo que fue a partir de un problema familiar. Ahí comenzó a subir y bajar la presión", "Por la situación económica, el gobierno, la inseguridad, las injusticias".

La categoría "biológica" agrupó aquellas creencias referidas a los factores de riesgo no modificables, asociados con aspectos fisiológicos, tales como: genética, herencia, envejecimiento, menopausia, entre otros. Algunas respuestas de este tipo fueron: "Según tengo entendido, y por las explicaciones del médico, mi enfermedad es hereditaria", "Se lo atribuyo a la edad, eso es lo principal", "Creo que hay predisposición genética ya que mi mamá falleció de un accidente cerebrovascular".

En relación a la comparación de las variables afrontamiento a la enfermedad y estilo de vida en relación a la salud, según el tipo de creencias y el género, los resultados del cálculo MANOVA arrojaron valores T y F de Hotelling y de probabilidad de error, que indican que las creencias se asociaron significativamente con el afrontamiento (THott=.14; FHott $(8 ; 178)=3.13 ; p=.00)$ pero no con el estilo de vida en relación a la 
salud (THott=.05; FHott $(6 ; 180)=1.44 ; p=.20)$; el género se asoció significativamente con el afrontamiento ante la hipertensión (THott=.09; FHott $(8 ; 178)=1.96 ; p=.05)$ y también con los hábitos saludables (THott=.23; FHott (6;180)=6.7; p=.00). Asimismo, cabe señalar que ambos factores (creencias y género) no interactuaron significativamente, tanto en el caso del afrontamiento (THott=.02; FHott $(8 ; 178)=.41 ; \mathrm{p}=.91$ ) como en el del estilo de vida (THott=.02; FHott $(6 ; 180)=.57 ; p=.75)$.

La tabla 1 muestra los puntajes promedios obtenidos por los pacientes en las variables afrontamiento y estilo de vida relacionado con la salud, según el grupo de sujetos: creencias de tipo psicosociales y creencias de tipo biológicas. Asimismo, se indican los resultados del MANOVA, presentándose los valores de T y F de Hotelling correspondientes a interrelaciones simultáneas de las creencias y las ocho estrategias de afrontamiento y, por otra parte, de las creencias y las seis dimensiones del estilo de vida. Finalmente, los valores F para cada ítem que compone el afrontamiento y las conductas en relación a la salud, y los valores " $p$ " de probabilidad de error para cada uno de ellos.

Tabla 1. Afrontamiento y estilo de vida según tipo de creencia

\begin{tabular}{|c|c|c|c|c|c|c|}
\hline \multicolumn{7}{|c|}{ Creencia sobre principal causa de HTA } \\
\hline & \multicolumn{2}{|c|}{ Psicosocial } & \multicolumn{2}{|c|}{ Biológica } & & \\
\hline & $n$ & $\%$ & $n$ & $\%$ & & \\
\hline & 120 & 63.5 & 69 & 36.5 & \multicolumn{2}{|l|}{ MANOVA } \\
\hline & & & & & \multicolumn{2}{|c|}{$\begin{array}{l}\text { FHott }(8 ; 178)=3.13 \\
\quad p=.00\end{array}$} \\
\hline Estrategias de afrontamiento & $m$ & de & $m$ & de & $F(1 ; 185)$ & $\boldsymbol{p}$ \\
\hline Resolución planificada problemas & 3.2 & .82 & 3.5 & .76 & 5.3 & .02 \\
\hline Reconsideración positiva & 3.1 & .85 & 3.1 & .88 & .16 & .69 \\
\hline Confrontación & 3 & .67 & 3 & .62 & .12 & .73 \\
\hline Búsqueda apoyo social & 2.9 & .9 & 3 & 1 & .03 & .86 \\
\hline Autocontrol & 2.7 & .66 & 2.7 & .66 & .12 & .74 \\
\hline Aceptación responsabilidad & 2.8 & .90 & 2.4 & .78 & 11.7 & .00 \\
\hline Distancia & 2.4 & .79 & 2.5 & .82 & .95 & .33 \\
\hline \multirow[t]{3}{*}{ Escape, evitación } & 2.2 & .63 & 2.2 & .63 & .74 & .39 \\
\hline & & & & & \multicolumn{2}{|l|}{ THott=.05 } \\
\hline & & & & & \multicolumn{2}{|c|}{$\begin{array}{l}\text { FHott }(6 ; 180)=1.44 \\
\quad p=.20\end{array}$} \\
\hline $\begin{array}{l}\text { Dimensiones del estilo de vida } \\
\text { relacionado con la salud }\end{array}$ & $m$ & de & $m$ & de & $F(1 ; 185)$ & $p$ \\
\hline Nutrición & 3.2 & .62 & 3.4 & .67 & 4.2 & .04 \\
\hline Actividad física & 2.3 & .94 & 2.5 & 1.1 & 3 & .09 \\
\hline Autorrealización & 3.5 & .66 & 3.6 & .69 & .79 & .38 \\
\hline Relaciones interpersonales & 3.5 & .68 & 3.6 & .72 & .40 & .53 \\
\hline Responsabilidad con la salud & 3.2 & .69 & 3.4 & .71 & 5.3 & .02 \\
\hline Manejo del estrés & 2.8 & .69 & 3 & .73 & 4.3 & .04 \\
\hline
\end{tabular}


El significado respecto de la enfermedad es una construcción social, en la cual se reflejan los valores y las normas culturales. Las creencias sobre el proceso salud-enfermedad constituyen una expresión de las prácticas, de la cultura y de los valores de los grupos sociales (León et al. 2003).
Respecto a la variable afrontamiento, los resultados de la tabla 1 muestran que existen diferencias estadísticamente significativas entre los grupos con distintas creencias, en dos estrategias de afrontamiento. El grupo de pacientes que creen que la HTA estaría causada por factores biológicos, utilizaron, en mayor medida, la estrategia "Resolución planificada de problemas", mientras que el grupo que respondió que su HTA estaría provocada por factores psicosociales, utilizó, más frecuentemente, la estrategia "Aceptación de la responsabilidad".

En cuanto a la variable estilo de vida relacionado con la salud, los valores $T$ y $F$ de Hotelling informan que no es significativo el efecto de las creencias sobre dicha variable. No obstante, los valores $F$ para cada dimensión de la misma, indican que existen diferencias estadísticamente significativas entre los dos tipos de creencias, en tres de ellas: "Nutrición", "Responsabilidad con la salud" y "Manejo del estrés". Al respecto, resultaría probable que el género influya en este resultado, dado que, tal como fue informado anteriormente, los valores de T y $F$ de Hotteling arrojados por el MANOVA, resultaron significativos, sin que el género y las creencias se relacionen entre sí.

En las tres dimensiones, fue el grupo con creencias de tipo biológicas sobre las causas de la HTA, el que mostró un puntaje más alto, es decir, presentó un estilo de vida más saludable. Algunos de los ítems que conforman estas dimensiones, y que reflejan dichas conductas saludables, son los siguientes. Nutrición: "Tomo el desayuno diariamente", "Observo las etiquetas nutritivas de los alimentos envasados para identificar el contenido de sodio y grasas", "Como entre tres y cinco porciones de vegetales todos los días", "Como entre dos y cuatro porciones de frutas diariamente". Responsabilidad con la salud: "Le informo a un médico o a otros profesionales de la salud cuando tengo algún síntoma extraño", "Si es necesario, le hago preguntas a los profesionales de la salud para entender sus indicaciones", "Busco una segunda opinión cuando tengo dudas sobre las recomendaciones de un médico", "Examino mi cuerpo para identificar cambios físicos o señales peligrosas". Manejo del estrés: "Duermo lo suficiente", "Me dedico algún tiempo para relajarme, todos los días", "Acepto aquellas cosas en mi vida que no puedo cambiar", "Me concentro en pensamientos agradables a la hora de dormir".

\section{Discusión}

El significado respecto de la enfermedad es una construcción social, en la cual se reflejan los valores y las normas culturales. Las creencias sobre el proceso saludenfermedad constituyen una expresión de las prácticas, de la cultura y de los valores de los grupos sociales (León et al. 2003).

En el presente trabajo, que indaga sobre la principal causa que los pacientes le atribuyen a su HTA, se ha evidenciado que las explicaciones se categorizan en dos tipos: causas de origen psicosocial y causas de origen biológico. Las causas de origen psicosocial estuvieron conformadas por aquellas respuestas que aludieron a cuestiones tales como las emociones, la personalidad, los conflictos vinculares, el estrés laboral, los duelos, entre otros. Por su parte, los sujetos que se refirieron a los factores biológicos como los principales desencadenantes de la enfermedad, mencionaron factores como la herencia, la menopausia y la edad. Siguiendo la revisión de León et al. (2003) se puede observar que los elementos que surgieron en el presente estudio se repiten en diversas sociedades, y conforman los sistemas explicativos que elaboran los pacientes sobre las causas de su salud y enfermedad. 
Pág 122

Los resultados revelan que el grupo de pacientes que manifestó como causas de la HTA las creencias de tipo biológico, habrían evaluado la enfermedad como un problema con más posibilidades de transformación, en comparación con aquellos que respondieron con creencias de índole psicosocial.
Entre las dos categorías, predominaron las causas de tipo psicosocial, hecho coincidente con trabajos previos que muestran que los aspectos psicosociales son el tipo de causas mayormente referidas por los pacientes (Arrieta-Pérez \& GarfíasJiménez, 2010; Beune, Haafkens, Schuster, \& Bindels, 2006; Correa Sánchez, Arrivillaga Quintero, \& Varela Arévalo, 2004; García de Alba-García et al., 2012; Granados, Roales-Nieto, Moreno, \& Ybarra, 2006). De este modo, se advierte sobre la importancia de los aspectos psicosociales en el enfermar, y el gran peso que éstos tienen en las creencias de los pacientes hipertensos. En el trabajo de Beune et al. (2006), los sujetos diferenciaron una HTA "natural“ de otra „creada“. La HTA natural fue aquella atribuida a causas biológicas y la HTA creada fue la consecuencia de situaciones de tipo psicosocial, especialmente el estrés. En dicho trabajo, también se observó que los pacientes refirieron, en mayor medida, que su HTA no era natural, sino producto de problemáticas cotidianas.

Los datos del presente estudio apoyan parcialmente la hipótesis formulada.

Por un lado, en lo que se refiere a las estrategias de afrontamiento a la enfermedad, éstas difieren entre los pacientes hipertensos, en función del tipo de creencias sobre las causas de la enfermedad.

Al respecto, Lazarus y Folkman (1986) sostienen que cuando el acontecimiento es percibido como modificable, los individuos tienden a alterarlo, mientras que cuando consideran que tienen pocas posibilidades de transformarlo, intentan regular la emoción que les produce. Desde esta perspectiva, los resultados revelan que el grupo de pacientes que manifestó como causas de la HTA las creencias de tipo biológico, habrían evaluado la enfermedad como un problema con más posibilidades de transformación, en comparación con aquellos que respondieron con creencias de índole psicosocial. Una posible interpretación responde a que los factores biológicos, en tanto causas, constituyen una entidad más tangible que los factores psicosociales, con indicadores más concretos, lo cual favorecería un comportamiento más activo y asertivo al momento de afrontar la enfermedad.

La relación entre el tipo de atribuciones causales y el modo de afrontamiento o comportamientos afines, son complejas. La revisión de Hall, French y Marteau (2003) sobre 65 estudios, tuvo por objetivo analizar las relaciones entre las creencias sobre las causas de una amplia variedad de enfermedades, mayormente crónicas, y los comportamientos adaptativos. Sus hallazgos evidenciaron que las asociaciones entre ambos factores no eran consistentes, ni coherentes, en la generalidad de los casos. Por su parte, el metaanálisis realizado por Hagger y Orbell (2003) halló patrones lógicos y previsibles de asociación entre los dominios referidos a la identidad, el control, la curación, las consecuencias, el período temporal de la enfermedad y las estrategias de afrontamiento empleadas. Observaron que aquellos individuos que interpretaron la enfermedad de manera más favorable, positiva y refiriendo un mayor control sobre la misma, fueron quienes presentaron un mayor grado de bienestar psicológico y utilizaron las estrategias de afrontamiento más activas, centradas en el problema, y de reevaluación positiva. No obstante, Morrison y Bennett (2008) sostienen que dicho estudio no tuvo en cuenta la dimensión de las causas de la enfermedad, por resultar complicada y problemática, debido al posible solapamiento generado por las múltiples atribuciones referidas por los pacientes.

Retomando la hipótesis formulada, y en lo que respecta a la variable estilo de vida, si bien los resultados del estudio indicaron que, de manera global, no existen dife- 
Pág 123

Si bien las creencias sobre la enfermedad reflejan la información provista por el médico, a veces dichas creencias exceden este tipo de información, ya que sucede que la información médica se combina con las interpretaciones de los pacientes sobre sus vivencias subjetivas, los valores culturales, el conocimiento de sentido común, entre otros (Torres-López, 2002). rencias entre los distintos grupos de creencias en esta variable, al tener en cuenta cada dimensión de manera independiente sí se observaron diferencias en algunas de ellas. Se propone, por lo tanto, proseguir con el estudio, aumentando la muestra de pacientes hipertensos, para analizar qué otras variables socioculturales probablemente esclarezcan los resultados.

Por lo mencionado, se destaca la importancia de continuar indagando sobre esta problemática en futuras investigaciones, a fin de profundizar en el estudio de las creencias que elaboran los pacientes sobre las causas de la enfermedad, como uno de los posibles elementos predictores de la adherencia y las conductas en salud.

Es fundamental señalar que se deben tener en cuenta las características particulares de la HTA, tales como el carácter asintomático y silente, que coadyuvan en la construcción de las creencias y en las conductas que presentan los pacientes. El rol del médico, en tanto una clara explicación y advertencia de estas particularidades de la HTA, colabora para la correcta evaluación del paciente sobre el riesgo para su salud. En general, los pacientes perciben, equívocamente, una sintomatología de la HTA, y a partir de ese preconcepto asumen, erróneamente, que podrían detectar la suba de la presión. Esta confusión genera a menudo el abandono del tratamiento por los pacientes, que actúan como si se tratara de una enfermedad aguda, en vez de crónica (Granados Gámez \& Roales Nieto, 2007; Granados Gámez, Roales-Nieto, Moreno, \& Ybarra, 2007).

Si bien las creencias sobre la enfermedad reflejan la información provista por el médico, a veces dichas creencias exceden este tipo de información, ya que sucede que la información médica se combina con las interpretaciones de los pacientes sobre sus vivencias subjetivas, los valores culturales, el conocimiento de sentido común, entre otros (Torres-López, 2002). Por ello, es necesario que el médico se capacite y fortalezca sus habilidades comunicacionales; de este modo, estaría entrenado para tomar en cuenta, por ejemplo, las creencias, hábitos y comportamientos de los pacientes, a fin de actuar de la mejor manera posible en el tratamiento y la atención (Buendía, 2012; Coca, Gómez, Llisterri, \& Camafort, 2012; Feltri et al., 2015; GutiérrezAngulo, Lopetegi-Uranga, Sánchez-Martín, \& Garaigordobil-Landazabal, 2012; Viego \& Luján Temporelli, 2016; Zambrano, Duitama, Posada, \& Flórez, 2012).

El panorama mencionado compromete, como una necesidad imperiosa, a realizar un abordaje integral de este tipo de enfermedades crónicas, que incluya la consideración de los aspectos tanto cognitivos como comportamentales, con técnicas novedosas, participativas y críticas (Holguín, Correa, Arrivillaga, Cáceres, \& Varela, 2006).

Si bien se deben considerar las debilidades metodológicas propias de este tipo de investigaciones, se destaca su contribución al ámbito de la Psicología de la Salud. Entre sus aportes, se encuentra el de proporcionar elementos de juicio para el diseño y planificación de dispositivos psicoeducativos dirigidos a la comunidad. De este modo, si los profesionales de la salud comprenden y reconocen los factores psicosociales asociados a las conductas en salud, dispondrán de otro elemento que les permita trabajar en la adherencia al tratamiento (Buendía, 2012; Coca, et al., 2012; Feltri et al., 2015; Granados Gámez \& Roales-Nieto, 2005; Gutiérrez-Angulo et al., 2012; Viego \& Luján Temporelli, 2016; Zambrano et al., 2012).

En síntesis, y en función de lo anteriormente expuesto, algunos aspectos a incorporar y tener en cuenta son: 
- Generar un espacio propicio para el abordaje interdisciplinario de diversos profesionales (cardiólogos, psicólogos, nutricionistas, entre otros). Tal como se ha señalado, la HTA es un trastorno que se debe abordar desde diversas perspectivas.

- Al momento de realizar un abordaje integral, tomar en consideración las variables psicosociales como el afrontamiento hacia la enfermedad, las creencias del paciente y el estilo de vida, entre otros, valorando las preocupaciones y los conocimientos previos con los que llegan los pacientes a la consulta.

- Por lo mencionado, resulta necesario incluir, en la evaluación global, datos sobre el contexto sociocultural, en particular el género y las vivencias de los pacientes.

\section{Referencias}

Ajzen, I., \& Fishbein, M. (1980). Understanding attitudes and predicting social behavior. Nueva Jersey (NJ): Prentice-Hall.

Álvarez L., Rueda Z., González L., \& Acevedo L. (2010). Promoción de actitudes y estrategias para el afrontamiento de la diabetes mellitus y la hipertensión arterial en un grupo de enfermos crónicos de la ciudad de Bucaramanga. Psicoperspectivas, 9(2), 279-290. http://www.scielo.cl/pdf/psicop/v9n2/art13.pdf

Arrieta-Pérez, R. T., \& Garfías-Jiménez, F. (2010). Creencias sobre el origen de los factores de riesgo para padecer hipertensión arterial en un grupo de pacientes hipertensos mexicanos. Archivos en Medicina Familiar, 12(2), 57-64. http://new. medigraphic.com/cgi-bin/resumen.cgi?IDARTICULO=26900

Bernal Trujillo, C., Lemos Hoyos, M., Medina Durango, l. E., Ospina Duque, J., \& Torres Y. (2009). Estrategias de afrontamiento y presión arterial. Revista CES Psicología, 2(2), 49-59. http://revistas.ces.edu.co/index.php/psicologia/article/view/896/589

Beune, E. J., Haafkens, J. A., Schuster, J. S., \& Bindels, P. J. (2006). "Under pressure": how Ghanaian, African-Surinamese and Dutch patients explain hypertension. Journal of Human Hipertensión, 20(12), 946-955. http://www.nature.com/ihh/index.html

Buendía, J. A. (2012). Actitudes, conocimientos y creencias del paciente hipertenso sobre la medicación antihipertensiva. Biomédica, 32(4), 578-584. http://www.revistabiomedica.org/index.php/biomedica/article/view/421

Castañeda, M. B., Cabrera, A. F., Navarro, Y., \& de Vries, W. (2010). Procesamiento de datos y análisis estadísticos utilizando SPSS. Un libro práctico para investigadores y administradores educativos. Porto Alegre: EDIPUCRS. http://www.pucrs.br/edipucrs/spss.pdf

Coca, A., Gómez, P., Llisterri, J. L., \& Camafort, M. (2012). Riesgo cardiovascular percibido por el paciente hipertenso y grado de cumplimiento terapéutico en hipertensión arterial: estudio Cumplimenhta. Hipertensión y Riesgo Vascular, 29(4), 136-144. Elsevier

Cordero, A., Bertomeu-Martínez, V., Mazón, P., Fácila, L., Bertomeu-González, V., Cosín, J., ... González-Juanatey, J. R. (2011). Factores asociados a la falta de control de la hipertensión arterial en pacientes con y sin enfermedad cardiovascular. Revista Española de Cardiología, 64(7), 587-593. http://www.revespcardiol.org/es/ factores-asociados-falta-control-hipertension/articulo/90023521/

Correa Sánchez, D., Arrivillaga Quintero, M., \& Varela Arévalo, M. T. (2004). Conocimientos y creencias sobre la hipertensión arterial presentes en usuarios de servicios de salud. Pensamiento Psicológico, 3, 41-58. http://revistas.javerianacali. edu.co/index.php/pensamientopsicologico/article/view/2

Corugedo Rodríguez, M. C., Martín Alfonso, L., \& Bayarre Vea, H. (2011). Adherencia terapéutica en pacientes con hipertensión arterial del Policlínico Universitario “Manuel Fajardo" en el Municipio Cruces, Cienfuegos, 2009. Revista Cubana de Medicina General Integral, 27(4), 504-512. SciELO 
Edwards, J., \& O Neill, R. M. (1998). The Construct Validity of Scores on the Ways of Coping Questionnaire: Confirmatory Analysis of Alternative Factor Structures. Educational and Psychological Measurement, 58(6), 955-983. http://epm.sagepub.com/content/58/6/955.abstract

Espinosa García, J., Martell Claros, N., Llerena Ruiz, A., \& Fernández Bergés Gurrea, D. (2012). Cumplimiento farmacológico en el tratamiento de la hipertensión arterial. Revisión de los estudios publicados entre los años 1975 y 2011. Semergen, 38(5), 292-300. Elsevier

Feltri, A., Ventura, C., Gallucci, E., Quiroga, J. M., Allevato, J., Bavio, E., ... Olavegogeascoechea P. A. (2015). Percepción de enfermedad en pacientes con diabetes tipo 2 e hipertensión arterial. Estudio piloto. Revista de la Federación Argentina de Cardiología, 44(3), 164-169. http://www.fac.org.ar/2/revista/15v44n1/opinion/ opinion01/piskorz.php

Fleiss, J. L., Levin, B., \& Paik, M. C. (2004). Statistical methods for rates and proportions (3ra ed.). New York: Wiley.

Folkman, S., Lazarus, R., Pimley, S., \& Novacek, J. (1987). Age differences in stress and coping process. Psychology and Aging, 2(2), 171-184. https://www.ncbi.nlm. nih.gov/pubmed/3268206

García de Alba-García, J. E., Salcedo-Rocha, A. L., Vargas-Guadarrama, L. A., \& García de Alba-Verduzco, J. E. (2012). La antropología cognitiva aplicada al estudio de las causas de la hipertensión arterial en Guadalajara, Jalisco, México. Cirugía y Cirujanos, 80(3), 247-252. http://new.medigraphic.com/cgi-bin/resumen. cgi?IDARTICULO=35343

Gómez LLambí, H., \& Piskorz, D. (2013). Hipertensión arterial, epidemiología, fisiología, fisiopatología, diagnóstico y terapéutica. Buenos Aires: Inter-Médica.

Granados, G., Roales-Nieto, J. G., Moreno, E., \& Ybarra, J. L. (2006). Creencias y conductas de corrección en pacientes con hipertensión arterial. International Journal of Clinical and Health Psychology, 8(3), 705-718. http://www.redalyc.org/ pdf/337/33712016006.pdf

Granados Gámez, G., \& Roales-Nieto, J. G. (2005). Formación de creencias sobre falsos síntomas en pacientes con hipertension. International Journal of Psychology and Psychological Therapy, 5(2), 165-206. http://www.ijpsy.com/volumen5/ num2/118/formacin-de-creencias-sobre-falsos-sntomas-ES.pdf

Granados Gámez, G., \& Roales Nieto, J. G. (2007). Creencias relacionadas con la hipertensión y adherencia a los diferentes componentes del tratamiento. International Journal of Psychology and Psychological Therapy, 7(3), 393-403. http:// www.ijpsy.com/volumen $7 /$ num $3 / 177 /$ creencias-relacionadas-con-la-hipertensi-ES.pdf

Granados Gámez, G., Roales Nieto, J. G., Moreno, E., \& Ybarra, J. L. (2007). Creencias en síntomas y adherencia al tratamiento farmacológico en pacientes con hipertensión. International Journal of Clinical and Health Psychology, 7(3), 697-707. http://www.redalyc.org/pdf/337/33770308.pdf

Grimson, W. R., De Blanco, A. C., Estrugamou, M., Lastres, E., Necchi, S., \& Phillpott, E. (1972). Investigación epidemiológica en entidades psiquiátricas. Boletín de la Oficina Sanitaria Panamericana, 79, 572.

Guevara-Gasca, M. P., \& Galán-Cuevas, S. (2010). El papel del estrés y el aprendizaje de las enfermedades crónicas: Hipertensión arterial y Diabetes. Revista Latinoamericana de Medicina Conductual, 1(18), 47-55. http://www.redalyc.org/ pdf/2830/283021975006.pdf

Gutiérrez-Angulo, M. L., Lopetegi-Uranga, P., Sánchez-Martín I., \& GaraigordobilLandazabal, M. (2012). Cumplimiento terapéutico en pacientes con hipertensión arterial y diabetes mellitus 2. Revista de Calidad Asistencial, 27(2), 72-77. Elsevier 
Hagger, M. S., \& Orbell, S. (2003). A Meta-analytic review of the common-sense model of illness representations. Psychology \& Health, 18(2), 141-184. Researchgate

Hall, S., French, D. P., \& Marteau, T. M. (2003). Causal Attributions Following Serious Unexpected Negative Events: A Systematic Review. Journal of Social and Clinical Psychology, 22, 515-536. https://kclpure.kcl.ac.uk/portal/en/publications/causal-attributions-following-serious-unexpected-negative-events-a-systematic-review(aaf672dd-5a314667-8b5a-ce2876ae599d)/export.html

Holguín, L., Correa, D., Arrivillaga, M., Cáceres, D., \& Varela, M. (2006). Adherencia al tratamiento de hipertensión arterial: efectividad de un programa de intervención biopsicosocial. Universitas Psychologica, 5(3), 535-547. http://www.redalyc. org/pdf/647/64750309.pdf

Lazarus, R. S., \& Folkman, S. (1986) Estrés y procesos cognitivos. Barcelona: Martínez Roca. Leibovich de Figueroa, N. B., \& Schufer, M. L. (2002). El "malestar" y su evaluación en diferentes contextos. Buenos Aires: Eudeba.

León, M., Páez, D., \& Díaz, B. (2003). Representaciones de la enfermedad. Estudios sociales y antropológicos. Boletín de Psicología, 77, 39-70.

Martín Alfonso L., Bayarre Vea H., La Rosa Matos Y., Orbay Araña M. C., Rodríguez Anaya J., Vento Iznaga R., ... Acosta González, M. (2007). Cumplimiento e incumplimiento del tratamiento antihipertensivo desde la perspectiva del paciente. Revista Cubana de Salud Pública, 33(3). http://scielo.sld.cu/scielo.php?script=sci arttext \&pid $=$ S0864-34662007000300013

Mora, \& Araujo, M. (2002). La estructura social de la Argentina: evidencias y conjeturas acerca de la estratificación actual. Serie Políticas Sociales, 59. Chile: CEPAL. Repositorio CEPAL

Morales Calatayud, F. (1999). La psicología en la atención primaria. En Morales Calatayud, F., Psicología de la salud. Conceptos básicos y proyecciones de trabajo (pp.100-101). La Habana: Editorial Científico Técnica.

Moreno, E., \& Gil Roales-Nieto, J. (2003). El modelo de creencias en salud: revisión teórica, consideración crítica y propuesta alternativa. International Journal of Psychology and Psychological Therapy, 3(1), 91-109. http://www.redalyc.org/ pdf/560/56030105.pdf

Morrison, V., \& Bennett, P. (2008). Psicología de la salud. Madrid: Pearson Prentice Hall.

Nerín, I. (2015). Género y salud: Estudiar las diferencias para mejorar los resultados. Revista Clínica Española, 215(7), 391-392. https://medes.com/publication/104560

Ofman S. D., Pereyra Girardi C. I., Cófreces P., \& Stefani D. (2014). Importancia de las diferencias por género en el afrontamiento a la enfermedad: el caso de la hipertensión arterial esencial. Apuntes de Psicología, 32(2), 161-170. http://www. apuntesdepsicologia.es/index.php/revista/article/viewFile/508/402

Organización Mundial de la Salud (OMS). (1998). Promoción de la salud. Glosario. Recuperado de http://www.msssi.gob.es/profesionales/saludPublica/prevPromocion/docs/glosario.pdf

Organización Mundial de la Salud (OMS). (2013). Información general sobre la hipertensión en el mundo. Recuperado de http://www.who.int/cardiovascular diseases/publications/global brief hypertension/es/

Ortiz Viveros, G. R., \& Ortega Herrera, E. (2011). Capacidad predictiva de la adherencia al tratamiento en los modelos socio-cognitivos de creencias en salud. Psicología y Salud, 21(1), 79-90. http://www.uv.mx/psicysalud/psicysalud-21-1/21-1/ Godeleva-Rosa-Ortiz-Viveros.pdf

Pender, N. J. (1982). Health promotion in nursing practice. Norwalk, CT: Appleton-Century-Crofts. 
Rodríguez Marin, J., Pastor, M. A., \& Lopez Roig, S. (1993). Afrontamiento, apoyo social, calidad de vida y enfermedad. Psicothema, 5(Supl.1), 349-372. http://www. psicothema.com/pdf/1148.pdf

Silva, M. E., Barbosa, L. D., Oliveira, A. D., Gouveia, M. T., Nunes, B. M., \& Alves, E. L. (2008). As representações sociais de mulheres portadoras de Hipertensão Arterial. Revista Brasileira de Enfermagem, 61(4), 500-507. http://www.scielo.br/ scielo.php?pid=S0034-71672008000400017\&script=sci abstract\&tlng=pt

Souza Minayo, M. C. (1997). Etapa de análisis o tratamiento del material. En Souza Minayo, M. C., El desafío del conocimiento: investigación cualitativa en salud (1era ed.), (pp.165-207). Buenos Aires: Lugar Editorial.

Torres-López, T. M. (2002). Una aproximación cualitativa al estudio de las enfermedades crónicas: las representaciones sociales. Revista Universidad de Guadalajara, 23. Researchgate

Viego, V. N., \& Luján Temporelli, K. (2016). Determinantes socioeconómicos y ambientales de la hipertensión arterial en población adulta de Argentina. Revista Cubana de Salud Pública, 42(1), 27-36. http://scielo.sld.cu/pdf/rcsp/v42n1/spu04116. $\underline{\mathrm{pdf}}$

Walker, S. N., Kerr, M. J., Pender, N. J., \& Sechrist, K. R. (1990). A Spanish language version of the Health-Promoting Lifestyle Profile. Nursing Research, 39(5), 26873. https://www.ncbi.nlm.nih.gov/pubmed/2399130

Walker, S. N., Sechrist, K. R., \& Pender, N. J. (1987). The Health-Promoting Lifestyle Profile: Development and psychometric characteristics. Nursing Research, 36(2), 76-81. https://www.ncbi.nlm.nih.gov/pubmed/3644262

Zambrano, R., Duitama, J. F., Posada, J. I., \& Flórez, J. F. (2012). Percepción de la adherencia a tratamientos en pacientes con factores de riesgo cardiovascular. Revista Facultad Nacional de Salud Pública, 30(2), 163-174. https://aprendeenlinea. udea.edu.co/revistas/index.php/fnsp/article/view/10239 